\title{
Gesellschaft für Phytotherapie e.V. startete Fortbildungsreihe 2017| 2018 für Ärzte und Apotheker
}

Nachdem 2015 / 2016 die ersten 4 Module der Fortbildungsreihe „Phytopharmaka und Phytotherapie“ erfolgreich durchgeführt wurden und 11 Teilnehmer ihr Zertifikat „Phytotherapeut“ (siehe Zeitschrift für Phytotherapie 2 (2017) erhielten, fand das 1. Modul der neuen Serie vom 31.3.-2.4.2017 wiederum im Lindner Sport \& Aktiv Hotel Kranichhöhe, Much / Bergisches Land statt, an dem 13 Teilnehmer begrüßt werden konnten.

Die neue Serie besteht wiederum aus 4 Modulen, in denen die wichtigsten Indikationen und deren wissenschaftlich gesicherter Einsatz in der täglichen Praxis besprochen werden.

Die wissenschaftliche Leitung des 1 . Moduls übernahm Frau Prof. Karen Nieber (Mitglied des wiss. Kuratoriums der GPT). Die ärztliche Leitung lag in den Händen von Frau Prof. Karin Kraft (Präsidentin der GPT). Für die Organisation war Frau Cornelia Schwöppe (Schatzmeisterin der GPT) verantwortlich. Durch erfahrene Referentinnen und Referenten aus Praxis und Wissenschaft wurden umfassende theoretische und praktische Kenntnisse zur Phytotherapie bei psychischen und rheumatischen Erkrankungen vermittelt. Auch der Einsatz von Phytopharmaka bei Schlafstörungen und bei Hauterkrankungen stand auf dem Programm.

\section{Was sind Phytopharmaka?}

Die Fortbildungsveranstaltung begann mit dem Einführungsvortrag „Phytopharmaka versus chemisch-synthetische Arzneimittel“ (Prof. Nieber). Moderne Phytopharmaka stellen Arzneimittel pflanzlichen Ursprungs dar und müssen entsprechend den europäischen gesetzlichen Grundlagen dieselben Anforderungen wie chemisch-synthetische Arzneimittel erfüllen. Dazu gehören die Gewährleistung der pharmazeutischen Qualität, die Unbedenklichkeit in der Anwendung und der Nachweis der therapeutischen Wirksamkeit. Im Unterschied zu den chemisch definierten Arzneimitteln, die in der Regel nur einen oder wenige Wirkstoffe enthalten, sind Phytopharmaka Vielstoffgemische. Die Standardisierung des Pflanzenextraktes sichert seine pharmazeutische Qualität und bildet die Voraussetzung für die Reproduzierbarkeit der Wirksamkeit eines Phytopharmakons von Charge zu Charge. An Hand von Beispielen wurde ihr Nutzen v.a. im Bereich der Behandlung subakuter, chronischer und altersbedingter Krankheitsprozesse diskutiert.

Anschließend wurde Einsatz von Phytopharmaka in der Psychiatrie vorgestellt. Die erfahrene Apothekerin Jutta Doebel (Apotheke im Erfstadt Center, Erfstadt) besprach sowohl neurobiologische Grundlagen als auch mögliche und gesicherte Wirkmechanismen von pflanzlichen Arzneimitteln bei Depression und Demenz. Sie erklärte an Hand praktischer Beispiele Vorteile aber auch Grenzen der Anwendung pflanzlicher Arzneimittel bei diesen Patienten und gab Tipps für die Beratung in der Apotheke und Arztpraxis. Der Nachmittag wurde mit dem Vortrag „Abgrenzung Phytopharmaka von Nahrungsergänzungsmitteln“ von Frau Dr. Rose Schraitle (BAH, Bonn) fortgesetzt. Trotz des etwas theoretischen Themas gab es hierzu viele interessante Fragen, da gerade dieser Punkt gegenwärtig große Beachtung findet.

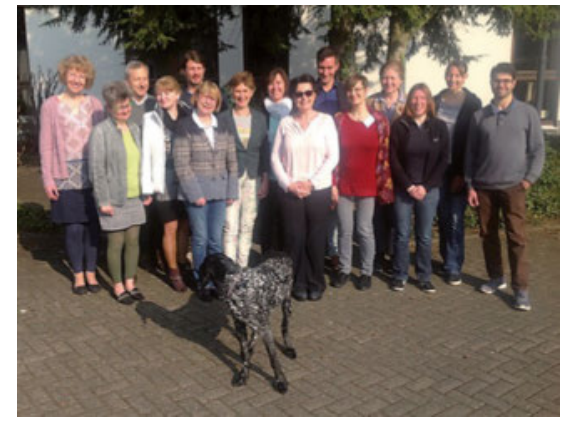

Bild der Teilnehmer des 1. Moduls mit einigen Referenten (c) Nieber

\section{Rheumatische Erkrankungen und Schlafstörungen}

Der zweite Tag begann mit dem Thema „Phytotherapie bei rheumatischen Erkrankungen“. In zwei Vorträgen referierte Frau Prof. Karin Kraft (Lehrstuhl für Naturheilkunde, Rostock) über die Anwendung von Interna und Externa bei verschiedenen rheumatischen Erkrankungen. Sowohl Wirkungsmechanismen als auch klinische Studien wurden vorgestellt. Die erfahrene Ärztin gab persönliche Empfehlungen für die Anwendung einzelner Präparate besonders hinsichtlich Dosierung und Anwendungsdauer und nannte Nebenwirkungen und Interaktionen. Sie resümierte, dass die Nebenwirkungen bei pflanzlichen Entzündungshemmern im Vergleich zu Synthetika deutlich geringer und weniger schwerwiegend sind, dass aber die schmerzlindernde Wirkung von Phytoanalgetika relativ schwach ist. In einer nachfolgenden Diskussionsrunde wurden in interaktiven Gesprächen mit den Teilnehmern diese Aspekte vertieft.

Der Nachmittag war dem Thema „Phytotherapie bei Schlafstörungen“ gewid- 
met. Oft fehlen Grundkenntnisse über Behandlungsmöglichkeiten und die negativen Folgen unbehandelter Schlafstörungen. Deshalb präsentierte Dr. Sebastian Michael (Löwen-Apotheke, Waldheim) in zwei Vorträgen anhand eines Entscheidungsbaums, wann und welche Phytopharmaka bei Schlafstörungen sinnvoll sind und wie sie angewendet werden sollten. Wirkungsmechanismen und neue Forschungsergebnisse ergänzten die Präsentationen. Der Referent betonte nachdrücklich, dass sich die volle Wirkung meist nicht sofort entfaltet. Die Präparate müssen mindestens für zwei Wochen eingenommen werden, am besten auch tagsüber.

\section{Fallbesprechungen}

Den nachfolgenden Workshop gestalteten Frau Apothekerin Dorothee Müssemeier (Alfter) und Frau Marita Kunkel, Fachärztin für Innere Medizin (Katholi- sche Kliniken Oberberg Herz-Jesu-Krankenhaus, Lindlar). Sie stellten Alltagspatienten mit rheumatischen Erkrankungen und Schlafstörungen vor und diskutierten gemeinsam mit den Teilnehmern, welche Erfahrungen sie bei der Umsetzung mit solchen Patienten haben und wie das in den vorherigen Vorträgen vermittelte Wissen in der Praxis umsetzbar ist. Der zweite Tag endete mit einem Vortrag zum Thema „Geschlechtsspezifische Unterschiede in der Pharmakotherapie“. Frau Prof. Karen Nieber erklärte pharmakokinetische und pharmakodynamische Unterschiede und stellt die Frage, ob dieses Thema auch für Phytopharmaka relevant ist und beachtet werden muss.

Am dritten Tag stand das Thema „Phytopharmaka in der Dermatologie“ auf der Agenda. Im ersten Teil besprach Dr. Sebastian Michael (Löwen-Apotheke, Waldheim), welche Arzneipflanzen und ihre Zubereitungen in der Dermatologie eingesetzt werden können. Der zweite
Teil beschäftigte sich dann mit einigen wichtigen Hauterkrankungen und der Frage „Wie gehe ich in der Praxis vor?“.

Die Fortbildungsveranstaltung war insgesamt sehr erfolgreich und durch intensive Diskussionen mit den Referenten und den Teilnehmern untereinander geprägt. In einem Abschlussgespräch wurde mehrheitlich ausgedrückt, dass sich die Teilnehmer bereits jetzt auf das 2. Modul freuen, das vom 15.-17.9.2017 wieder in Much/Bergisches Land stattfinden wird. Inhalte werden dann gastrointestinale Störungen, Harnwegsinfekte und Gefäßerkrankungen sein. Auch Vorträge zur Qualitätssicherung bei Phytopharmaka und die Stellung der Phytotherapie in Europa sind geplant.

Weitere Informationen zum 2. Modul finden sich demnächst auf der Homepage der GPT unter www.phytotherapie.de/ fortbildungsveranstaltungen.

Prof. Dr. Karen Nieber 